\title{
Síndrome PFAPA: Diagnóstico e Tratamento na Atenção Primária à Saúde
}

\author{
PFAPA Syndrome: Diagnosis and Treatment in Primary Health Care \\ Síndrome PFAPA: Diagnóstico y Tratamiento en la Atención Primaria a la Salud
}

Laniel Aparecido Bueno ${ }^{1}$, Débora Magalhães Paiva ${ }^{1}$, Paulo Henrique Pimenta de Carvalho ${ }^{1}$ (D)

${ }^{1}$ Universidade Estadual de Montes Claros (UNIMONTES). Montes Claros, MG, Brasil.

\section{Resumo}

Objetivo: Apresentar informações sobre o diagnóstico e tratamento da síndrome PFAPA na Atenção Primária à Saúde. Métodos: Revisão sistemática de literatura baseada na recomendação PRISMA e realizada nas bases de dados Scielo, Lilacs, Medline, IBECS e PubMed, incluindo estudos publicados no período de 2004 a 2018, além da consulta a outros documentos específicos da síndrome PFAPA. Resultados: Após busca e seleção, foram incluídos 31 artigos. Avaliação e Diagnóstico: A síndrome PFAPA acomete principalmente crianças, sendo caracterizada por febre periódica acompanhada por faringite, estomatite aftosa e/ou adenite cervical. Seu diagnóstico é clínico e por exclusão, baseado em critérios estabelecidos. Recomendações: Os episódios costumam responder a prednisona e, em graus variáveis, a cimetidina e colchicina. Casos refratários e acompanhados de hipertrofia tonsilar são candidatos a tonsilectomia, devendo ser encaminhados à avaliação otorrinolaringológica.

Palavras-chave: Febre Recorrente; Faringite; Estomatite Aftosa; Adenite; Atenção Primária à Saúde

\begin{abstract}
Objective: To present information about the diagnosis and treatment of PFAPA syndrome in Primary Health Care. Methods: Systematic review of literature based on the PRISMA recommendation and carried out in the Scielo, Lilacs, Medline, IBECS and PubMed databases, including studies published from 2004 to 2018, in addition to consulting other PFAPA syndrome specific documents. Results: After search and selection, 31 articles were included. Assessment and Diagnosis: PFAPA syndrome affects mainly children and is characterized by periodic fever accompanied by pharyngitis, aphthous stomatitis and/or cervical adenitis. Its diagnosis is clinical and by exclusion, based on established criteria. Recommendations: The episodes usually respond to prednisone and, in varying levels, cimetidine and colchicine. Refractory cases and accompanied by tonsillar hypertrophy are candidates for tonsillectomy, and should be referred to otorhinolaryngological evaluation.
\end{abstract}

Keywords: Relapsing Fever; Pharyngitis; Stomatitis, Aphthous; Lymphadenitis; Primary Health Care

Como citar: Bueno LA, Paiva DM, Carvalho PHP. Síndrome PFAPA: Diagnóstico e Tratamento na Atenção Primária à Saúde. Rev Bras Med Fam Comunidade. 2019;14(41):1815. https://doi.org/10.5712/rbmfc14(41)1815

\author{
Autor correspondente: \\ Laniel Aparecido Bueno. \\ E-mail: lanielbbueno@gmail.com \\ Fonte de financiamento: \\ declaram não haver. \\ Parecer CEP: \\ não se aplica. \\ Procedência e revisão por pares: \\ revisado por pares. \\ Recebido em: 11/07/2018. \\ Aprovado em: 10/12/2018.
}




\section{Resumen}

Objetivo: Presentar informaciones sobre el diagnóstico y tratamiento del síndrome PFAPA en la Atención Primaria a la Salud. Métodos: Revisión sistemática de literatura basada en la recomendación PRISMA y realizada en las bases de datos Scielo, Lilacs, Medline, IBECS y PubMed, incluyendo estudios publicados en el período 2004 a 2018, además de la consulta a otros documentos específicos del síndrome PFAPA. Resultados: Después de la búsqueda y selección, se incluyeron 31 artículos. Evaluación y Diagnóstico: El síndrome PFAPA acomete principalmente niños, siendo caracterizada por fiebre periódica acompañada por faringitis, estomatitis aftosa y/o adenitis cervical. Su diagnóstico es clínico y por exclusión, basado en criterios establecidos. Recomendaciones: Los episodios suelen responder a la prednisona y, en grados variables, a la cimetidina y colchicina. Los casos refractarios y acompañados de hipertrofia tonsilar son candidatos a tonsilectomía, debiendo ser encaminados a la evaluación otorrinolaringológica.

Palabras clave: Fiebre Recurrente; Faringitis; Estomatitis Aftosa; Linfadenitis; Atención Primaria de Salud

\section{Introdução}

Asíndrome Periodic Fever, Aphthous Stomatitis, Pharyngitis and Cervical Adenitis (PFAPA) foi descrita em 1987 por Marshall et al., sendo denominada síndrome de Marshall. Em 1989, essa designação foi alterada para o acrônimo FAPA e, posteriormente, para PFAPA, enfatizando a febre periódica, principal manifestação clínica. ${ }^{1}$

É caracterizada por episódios recorrentes de febre alta, acompanhada de estomatite aftosa, faringite e adenite cervical. ${ }^{2}$ Afeta principalmente crianças por volta dos 4 anos de idade, porém, o início dos sintomas pode ocorrer desde os primeiros meses de vida até os 12 anos. ${ }^{3}$ Embora mais comum na infância, a síndrome PFAPA vem sendo descrita em adultos nos últimos anos, manifestando-se na segunda década de vida, sendo o diagnóstico realizado na terceira década. ${ }^{4,5}$

A etiopatogênese da síndrome PFAPA ainda é desconhecida, havendo inúmeras hipóteses infecciosas e imunoinflamatórias. ${ }^{5-8}$

Estudos demonstram que pacientes com síndrome PFAPA possuem altos níveis de citocinas pró-inflamatórias e aumento de citocinas reguladoras da migração linfocitária. Isso sugere uma etiopatogênese imunoinflamatória, havendo desregulação imunológica com contínua ativação de citocinas pró-inflamatórias e redução de citocinas anti-inflamatórias. ${ }^{9}$

A síndrome PFAPA se assemelha com quadros de faringotonsilites, atendidas frequentemente na atenção primária, sendo necessário o diagnóstico diferencial entre essas condições.

O objetivo deste estudo é apresentar a síndrome PFAPA, abordando aspectos relacionados ao diagnóstico e tratamento em crianças e adultos na Atenção Primária à Saúde.

\section{Métodos}

Trata-se de um estudo de revisão sistemática de literatura baseado nos Preferred Reporting Items for Systematic Reviews and Meta-Analyses (PRISMA), sendo a busca dos estudos realizada nas bases de dados Scielo, Medline, Lilacs, PubMed e IBECS, além da consulta a dois manuais ${ }^{9,10}$ da Interamerican Association of Pediatric Otorhinolaryngology (IAPO) sobre a síndrome PFAPA e a inclusão dos critérios diagnósticos da síndrome PFAPA de Thomas et al. ${ }^{11}$ e Lawton. ${ }^{12}$ 
Na revisão sistemática foram utilizados os descritores "Febre Recorrente", "Faringite", "Estomatite Aftosa" e "Linfadenite" dos Descritores de Ciências da Saúde (DeCS) em português, inglês e espanhol para as bases SciELO, Lilacs, Medline e IBECS. A busca foi realizada pela associação dos descritores através do operador boleano AND nessas quatro bases de dados. Para a base PubMed foram utilizados os descritores apenas em inglês "Relapsing Fever", "Periodic fever", "Pharyngitis", "Stomatitis, Aphthous" e "Lymphadenitis" da Medical Subject Headings (MeSH) por meio da seguinte associação com os operadores boleanos OR e AND: "Relapsing Fever" OR "Periodic fever" AND "Pharyngitis" AND "Stomatitis, Aphthous" AND "Lymphadenitis".

A revisão foi realizada em março de 2018, tendo como critérios de inclusão artigos publicados entre 2004 a 2018, em inglês, português e espanhol, que abordassem o diagnóstico e tratamento da síndrome PFAPA. Os critérios de exclusão foram cartas a editores, teses, monografias, dissertações, artigos de opinião e estudos que abordassem apenas a patogênese da síndrome.

O nível de evidência científica dos estudos da revisão sistemática e outras referências consultadas foi classificado pelo Nível de Evidência por Tipo de Estudo - Oxford Centre for Evidence-Based Medicine.

\section{Resultados}

Na revisão sistemática, aplicando-se os critérios de inclusão, encontraram-se 124 artigos. Após a exclusão de artigos duplicados da mesma base de dados e entre bases distintas, alcançou-se o total de 99 artigos para a leitura dos resumos. Pela leitura dos resumos, 60 artigos foram excluídos por englobarem predominantemente a patogênese da síndrome PFAPA, atingindo 39 artigos para a leitura completa do texto. Após essa leitura, 8 artigos foram excluídos por não abordarem o diagnóstico e tratamento da síndrome PFAPA, chegando-se ao total de 31 artigos inclusos na revisão sistemática. Abaixo apresentamos as etapas de seleção dos artigos (Figura 1).

De acordo com a contribuição dos estudos quanto ao diagnóstico e/ou tratamento da síndrome PFAPA, a classificação dos níveis de evidência foi realizada de forma separada (Quadros 1 e 2).

\section{Avaliação e Diagnóstico}

A síndrome PFAPA é caracterizada por episódios de febre recorrente associada a estomatite aftosa, faringite e adenite, ocorrendo, frequentemente, por volta dos quatro anos de idade. ${ }^{3}$ Entretanto, as manifestações podem ocorrer desde os primeiros meses de vida até os 12 anos. ${ }^{3,6,13-15}$

A febre é de caráter recorrente a cada duas a 12 semanas, de $39^{\circ}$ a $40^{\circ} \mathrm{C}$, com duração de três a seis dias. Nos intervalos afebris o paciente encontra-se assintomático. ${ }^{5,14,16}$

Quanto à estomatite aftosa, as lesões se localizam predominantemente na região anterior da cavidade oral, são menores que $3 \mathrm{~mm}$, em pequenas quantidades e poupam as gengivas. Essas características permitem excluir outras condições que apresentam aftas, como a herpangina e a gengivoestomatite herpética. ${ }^{6,9,10,17}$

A adenite é uma manifestação frequente, de acometimento cervical, com gânglios pequenos, móveis e comumente a última manifestação a regredir. ${ }^{9,10,14}$ 


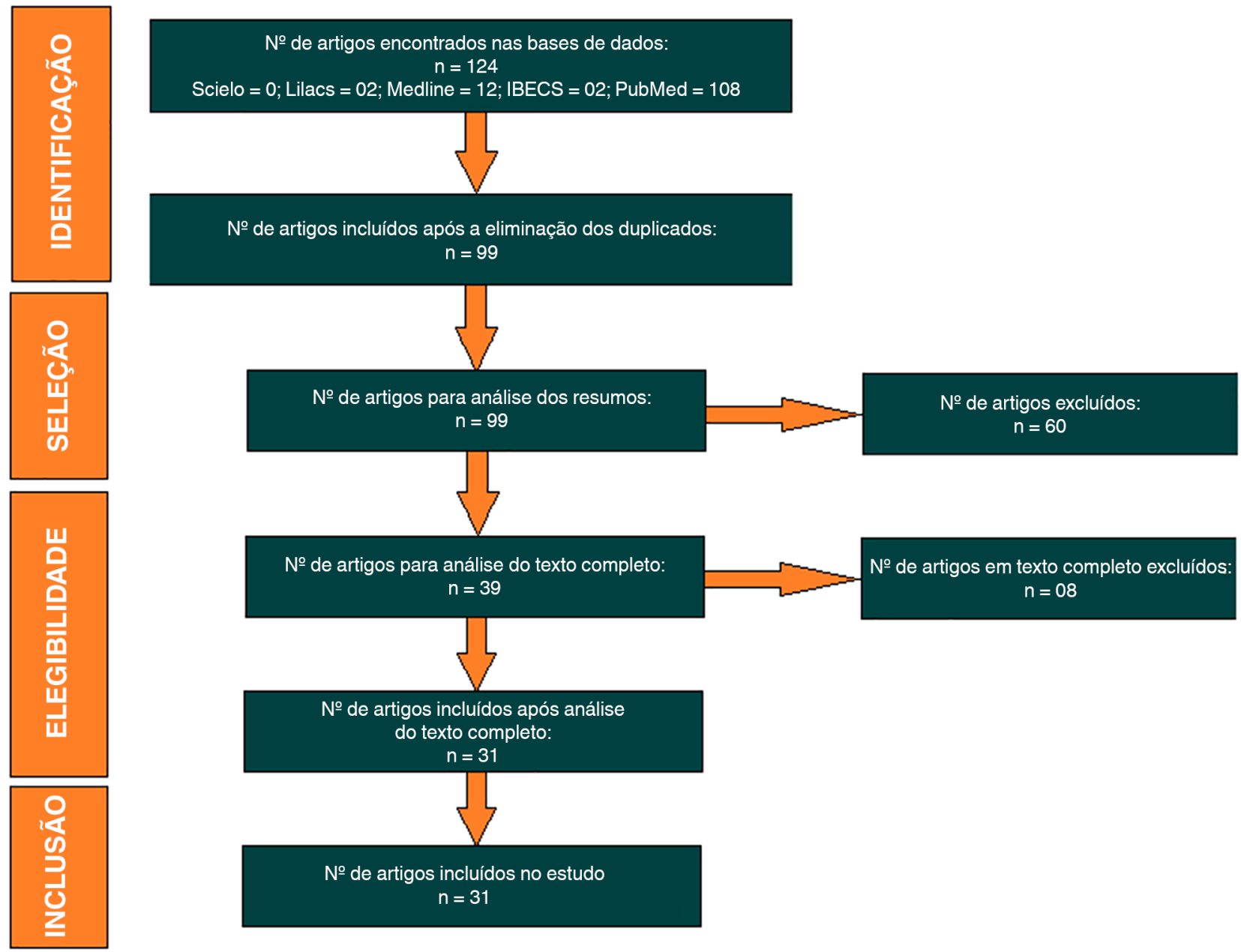

Figura 1. Fluxograma de seleção dos artigos conforme a recomendação PRISMA.

A faringite está presente em quase todos os pacientes, geralmente com hiperemia difusa nas regiões palatal e tonsilar, podendo ou não ser exsudativa. ${ }^{6,9,10,13-16,18,19}$

Além dessas manifestações típicas, existem outras atípicas, como dor abdominal, cefaleia, artralgia, mialgia, astenia, fadiga, erupção cutânea, úlceras genitais e edema facial. ${ }^{13,16,19-23}$

Geralmente, os pacientes não possuem infecção respiratória e mantêm-se saudáveis entre os episódios da síndrome, sem prejuízo nos processos de crescimento e desenvolvimento, no caso de crianças. ${ }^{6,15}$

O diagnóstico é baseado em critérios estabelecidos e exclusão de outros diagnósticos diferenciais. Assim, destacam-se os critérios diagnósticos de Thomas et al., ${ }^{11}$ modificados para os critérios de Lawton, ${ }^{12}$ que, por sua vez, apresenta os critérios de exclusão da síndrome ${ }^{15}$ (Quadros 3 e 4).

Os critérios de Thomas et al. ${ }^{11}$ apresentam alta sensibilidade, porém outras doenças são diagnósticadas errôneamente, o que torna importante os critérios de Lawton ${ }^{12}$ pelo caráter exclusivo dessas doenças. ${ }^{24}$

Os principais diagnósticos diferenciais da síndrome PFAPA encontrados com maior frequência na atenção primária são as faringotonsilites. São infecções autolimitadas restritas às tonsilas, faringe, úvula, palato mole e aos linfonodos do anel de Waldeyer. Vários microorganismos estão envolvidos: vírus, bactérias aeróbias (Streptococcus do grupo A) e anaeróbias, Mycoplasma, Toxoplasma e Candida sp. ${ }^{25}$ 
Quadro 1. Classificação do nível de evidência dos estudos em relação ao diagnóstico.

\begin{tabular}{|c|c|c|c|c|}
\hline Estudo por Primeiro Autor & Grau de Recomendação & Nível de Evidência & Base de Dados & Ano de Publicação \\
\hline Padeh et al. ${ }^{5}$ & $B$ & $2 B$ & PubMed & 2008 \\
\hline Fonseca et al. ${ }^{6}$ & B & $3 A$ & Lilacs & 2005 \\
\hline Silber ${ }^{9}$ & $\mathrm{D}$ & 5 & IAPO & 2007 \\
\hline Silber ${ }^{10}$ & $\mathrm{D}$ & 5 & IAPO & 2005 \\
\hline Thomas et al. ${ }^{11}$ & A & 1B & PubMed & 1999 \\
\hline Lawton $^{12}$ & A & $1 \mathrm{~B}$ & UpToDate & 2008 \\
\hline Tasher et al. ${ }^{13}$ & A & 1B & Medline & 2006 \\
\hline De Cunto et al. ${ }^{14}$ & C & 4 & PubMed & 2010 \\
\hline Sampaio e Marques ${ }^{15}$ & C & 4 & PubMed & 2011 \\
\hline Rigante et al. ${ }^{16}$ & A & $1 \mathrm{~B}$ & PubMed & 2016 \\
\hline Feder e Salazar ${ }^{17}$ & B & $2 B$ & PubMed & 2010 \\
\hline Król et al. ${ }^{18}$ & A & $1 \mathrm{~B}$ & Medline & 2013 \\
\hline Hofer et al..$^{19}$ & B & $2 B$ & PubMed & 2014 \\
\hline Scattoni et al. ${ }^{20}$ & B & $3 B$ & PubMed & 2014 \\
\hline Khodaghalian et al. ${ }^{21}$ & B & $3 B$ & PubMed & 2013 \\
\hline Li et al..$^{22}$ & B & 3B & PubMed & 2011 \\
\hline Cantarini et al. ${ }^{23}$ & B & $2 B$ & PubMed & 2012 \\
\hline Førsvoll et al. ${ }^{24}$ & B & $2 B$ & PubMed & 2012 \\
\hline Wurster et al. ${ }^{29}$ & B & $2 \mathrm{~B}$ & PubMed & 2011 \\
\hline Pinto et al. ${ }^{41}$ & B & $3 B$ & Medline & 2006 \\
\hline
\end{tabular}

Quadro 2. Classificação do nível de evidência dos estudos em relação ao tratamento.

\begin{tabular}{|c|c|c|c|c|}
\hline Estudo por Primeiro Autor & Grau de Recomendação & Nível de Evidência & Base de Dados & Ano de Publicação \\
\hline Lantto et al. ${ }^{7}$ & $B$ & $2 \mathrm{~B}$ & PubMed & 2016 \\
\hline Silber ${ }^{9}$ & D & 5 & IAPO & 2007 \\
\hline Silber ${ }^{10}$ & D & 5 & IAPO & 2005 \\
\hline Tasher et al. ${ }^{13}$ & C & 4 & Medline & 2006 \\
\hline Sampaio e Marques ${ }^{5}$ & B & $2 \mathrm{~B}$ & PubMed & 2011 \\
\hline Rigante et al. ${ }^{16}$ & A & $2 \mathrm{~A}$ & PubMed & 2016 \\
\hline Hofer et al. ${ }^{19}$ & B & $2 \mathrm{~B}$ & PubMed & 2014 \\
\hline Vanoni et al. ${ }^{26}$ & A & $1 \mathrm{~A}$ & PubMed & 2016 \\
\hline Butbul Aviel et al. ${ }^{27}$ & A & 1B & PubMed & 2016 \\
\hline Ciriza Barea et al. ${ }^{28}$ & C & 4 & IBECS & 2011 \\
\hline Wurster et al. ${ }^{29}$ & B & $2 B$ & PubMed & 2011 \\
\hline Dusser et al. ${ }^{30}$ & B & $2 B$ & PubMed & 2016 \\
\hline Tasher et al. ${ }^{31}$ & B & $2 \mathrm{~B}$ & PubMed & 2008 \\
\hline Stagi et al..$^{32}$ & B & $2 \mathrm{~B}$ & PubMed & 2014 \\
\hline Licameli et al. ${ }^{33}$ & B & 3B & PubMed & 2012 \\
\hline Erdogan et al. ${ }^{34}$ & B & 2B & PubMed & 2015 \\
\hline Garavello et al. ${ }^{35}$ & B & $2 \mathrm{~A}$ & PubMed & 2011 \\
\hline Pignataro et al. ${ }^{36}$ & B & 3B & PubMed & 2009 \\
\hline Garavello et al. ${ }^{37}$ & B & 3B & PubMed & 2009 \\
\hline Licameli et al. ${ }^{38}$ & C & 4 & PubMed & 2008 \\
\hline Wong et al. ${ }^{39}$ & B & $2 \mathrm{~B}$ & PubMed & 2008 \\
\hline Renko et al. ${ }^{40}$ & B & $2 \mathrm{~B}$ & Medline & 2007 \\
\hline
\end{tabular}


Quadro 3. Critérios diagnósticos de Thomas et al. ${ }^{11}$ (1999).

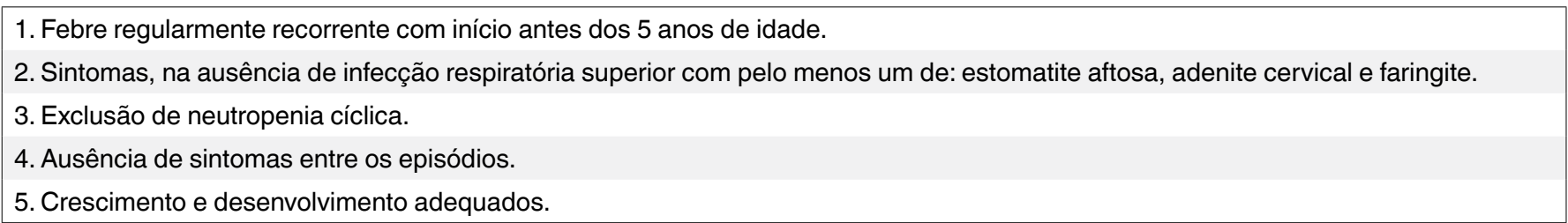
Fonte: Thomas et al. ${ }^{1}$

Quadro 4. Critérios diagnósticos modificados de Lawton (2008).

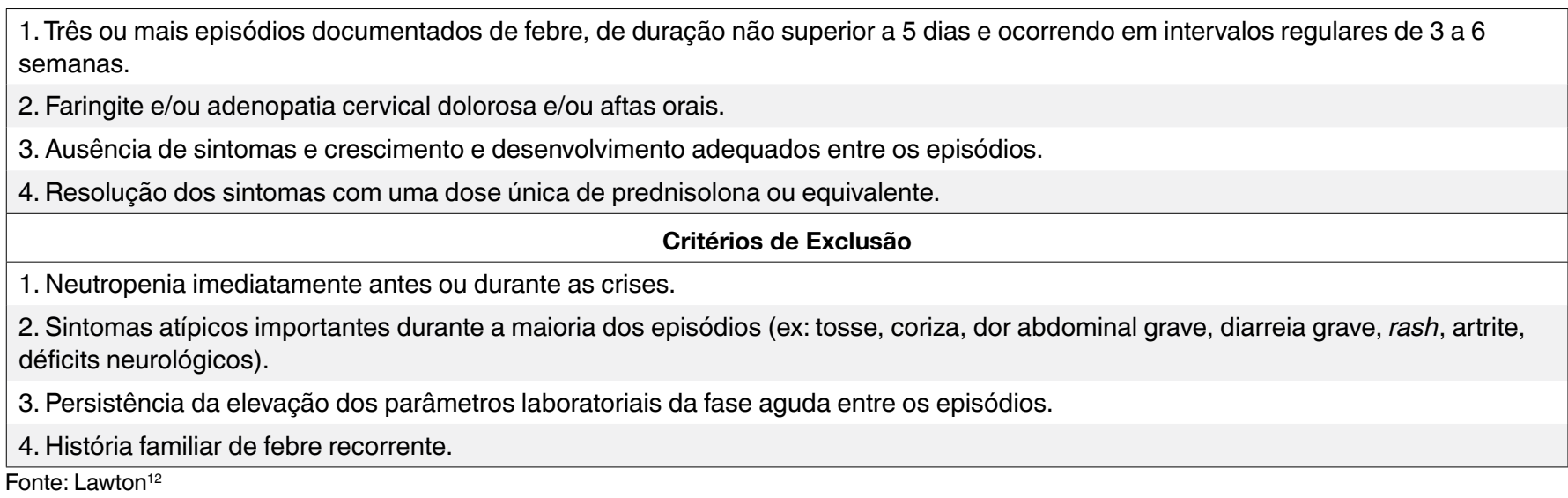

Cerca de $50 \%$ das faringotonsilites são virais, principalmente em crianças menores de dois anos. Destaque é feito para a herpangina, causada pelo vírus coxsakie A, com vesículas nas tonsilas, pilares anteriores, palato e faringe, que se rompem e formam pequenas aftas dolorosas. ${ }^{25}$ Embora essas lesões ocorram na síndrome PFAPA, possuem características distintas.

Nas faringotonsilites bacterianas, destaca-se o Streptococcus do grupo A. O pico de incidência é no inverno e primavera, acometendo crianças e adolescentes, sendo infrequente em menores de três anos. Apresenta instalação aguda de faringite, odinofagia, febre alta, cefaleia e dor abdominal. O exame físico revela hiperemia faringotonsilar; exsudato não é obrigatório e pode haver adenopatia cervical. ${ }^{25}$

Embora as faringotonsilites possuam características compatíveis com a síndrome PFAPA, essa tem maior número de episódios recorrentes do que as faringotonsilites. Não apresenta instalação insidiosa como as faringotonsilites virais. Acomete, na maior parte dos casos, crianças menores de cinco anos em comparação com a faringotonsilite bacteriana, que afeta frequentemente a faixa etária de 5 a 15 anos. Além disso, os episódios da síndrome PFAPA não ocorrem predominantemente no inverno, como as faringotonsilites.

Devemos considerar na suspeita de síndrome PFAPA pacientes com histórico de culturas de secreção de orofaringe negativas e antibioticoterapia para faringotonsilite bacteriana, sem resolução. ${ }^{22}$ Quando disponível, devemos solicitar cultura de secreção tonsilar em dois episódios diferentes e não sucessivos. .,10 $^{9}$

Embora mais prevalente na infância, estudos apontam a ocorrência da síndrome em adultos, sem predominância entre os gêneros, sendo uma hipótese diagnóstica principalmente em adultos com episódios inexplicados de febre recorrente. ${ }^{5,16}$ 
Em adultos, é comum a história de manifestações da síndrome na infância, com resolução e retorno na idade adulta por volta da segunda década de vida, ocorrendo o diagnóstico geralmente na terceira década. O quadro clínico é semelhante ao das crianças, porém com variações quanto ao número e duração dos episódios febris e na quantidade de manifestações associadas à febre, sendo que as crianças apresentam mais manifestações associadas aos episódios febris do que os adultos. ${ }^{5,16,23}$

\section{Recomendações}

O tratamento de escolha são os corticoides, especialmente a prednisona, por três a cinco dias. $\mathrm{O}$ período de melhora varia entre os estudos, porém ocorre após a administração da primeira ou segunda dose da medicação. Entretanto, um problema da corticoterapia é a redução do intervalo entre os episódios febris, além de não prevenir futuros episódios da síndrome PFAPA. Mesmo assim, o uso do corticoide apresenta ótimos resultados em muitos estudos. ${ }^{9,10,13,15,19,26-29}$

Dessa forma, o estudo de coorte internacional de Hofer et al. ${ }^{19}$ demonstrou rápida resolução dos episódios febris em 93 de 147 pacientes com síndrome PFAPA após dose única de corticoide, sendo que 46 pacientes apresentaram resposta parcial e oito não responderam à corticoterapia.

Estudo de coorte de Tasher et al. ${ }^{13}$ mostrou que em 51 de 54 pacientes com síndrome PFAPA houve rápida resolução dos episódios febris, em média de 10 horas, após a administração de uma dose única baixa de prednisona. Igualmente, Wurster et al. ${ }^{29}$ demonstraram, em uma coorte de 60 pacientes, que dos 44 indivíduos tratados com corticoide, 37 apresentaram resposta eficaz ao tratamento.

Porém, para os pacientes não respondedores à corticoterapia, uma alternativa é a cimetidina, que parece aumentar a síntese de inferferon, além de modular a liberação de citocinas e fator quimiotático de leucócitos envolvidos na síndrome PFAPA. ${ }^{9,15}$ Estudo de Wurster et al ${ }^{29}$ demonstrou no acompanhamento de 59 indivíduos de uma coorte de 60 pacientes com síndrome PFAPA, que, dos 25 tratados com cimetidina, seis apresentaram resultados eficazes na resolução dos episódios febris; seis pacientes obtiveram resultados moderados e 13 foram ineficazes.

A redução dos episódios da síndrome pode ser alcançada pela colchicina. ${ }^{16}$ Seu uso profilático é baseado nas semelhanças clínicas e laboratoriais entre a síndrome PFAPAe a febre familiar do mediterrâneo, além da experiência no tratamento dessa com a colchicina. Geralmente, é bem tolerada pelos pacientes. ${ }^{26,27,30}$

Aviel et al. ${ }^{27}$ mostraram em estudo controlado com colchicina redução significativa nos epsiódios febris em 18 pacientes com diagnóstico de síndrome PFAPA. Estudo observacional de Tasher et al. ${ }^{31}$ demonstrou, entre nove pacientes com episódios da síndrome tratados com colchicina, que oito apresentaram redução dos episódios, aumentando o intervalo de 1,7 para 8,4 semanas em média. Apenas um paciente não apresentou mudança na frequência dos episódios.

Outra opção é a suplementação de vitamina D. Muitos pacientes com síndrome PFAPA possuem níveis séricos baixos de vitamina $\mathrm{D}$ e sua suplementação reduz o número e a duração dos episódios da síndrome, como apresentado pelo estudo de caso-controle de Stagi et al., ${ }^{32}$ no qual os pacientes receberam suplementação de 400 UI de vitamina D, com redução dos episódios febris. Nesse estudo, a maioria dos pacientes possuíam níveis baixos de vitamina $D$, sugerindo a hipovitaminose $D$ como fator de risco para síndrome PFAPA. Entretanto, mais estudos são necessários para definir o papel da vitamina $D$ na terapêutica da síndrome PFAPA. ${ }^{26}$ 
Abaixo, apresentamos as medicações para a Síndrome PFAPA (Tabela 1).

Tabela 1. Medicações e posologias indicadas para a Síndrome PFAPA.

\begin{tabular}{lcc} 
Medicação & Posologias - Crianças & Posologias - Adultos \\
\hline Prednisona & 1 a $2 \mathrm{mg} / \mathrm{kg} / \mathrm{dia}$, por 3 a 5 dias & $20 \mathrm{mg} / \mathrm{dia}$, por 3 a $5 \mathrm{dias}$ \\
Cimetidina & 20 a $40 \mathrm{mg} / \mathrm{dia}, 1 \mathrm{a} 2$ vezes ao dia, por 6 a 8 meses & $200 \mathrm{mg} / \mathrm{dia}, 1$ a 2 veses ao dia, por 6 a $8 \mathrm{meses}$ \\
Colchicina & $0,5 \mathrm{a} 1,0 \mathrm{mg} / \mathrm{dia}$ & $0,5 \mathrm{a} 1,0 \mathrm{mg} / \mathrm{dia}$ \\
\hline
\end{tabular}

Outra abordagem em pacientes não responsivos à farmacoterapia e com hipertrofia de tonsilas é a tonsilectomia. Muitos estudos relatam redução do número de episódios ou até resolução completa da síndrome PFAPA com essa abordagem. ${ }^{7,26,29,33-39}$

Assim, Renko et al., ${ }^{40}$ em estudo randomizado envolvendo 26 pacientes com síndrome PFAPA, demonstraram que no grupo de caso de 14 pacientes submetidos a tonsilectomia houve desaparecimento imediato da síndrome. No grupo controle de 12 pacientes, a síndrome desapareceu espontaneamente em seis pacientes no período de seis meses.

Em estudo prospectivo, Licameli et al. ${ }^{33}$ acompanharam, por seis meses, 102 pacientes com síndrome PFAPA submetidos a tonsilectomia e verificaram resolução completa em 99 pacientes logo após a cirurgia; um paciente apresentou resolução seis meses mais tarde, um permaneceu apresentando episódios febris e um foi diagnosticado com outra doença.

\section{Encaminhamento}

Deve ser feito preferencialmente à otorrinolaringologia em casos de falha terapêutica e hipertrofia tonsilar.

Abaixo, apresentamos uma proposta de fluxograma para manejo da síndrome PFAPA (Figura 2).

\section{Discussão}

Aetiologia da síndrome PFAPAé desconhecida, havendo hipóteses infecciosas e imunoinflamatórias. ${ }^{5,6-8}$ Entretanto, estudos recentes apontam maior tendência imunoinflamatória em razão da recorrência de episódios idênticos. ${ }^{6,9,10,23}$

As citocinas mais elevadas durante os episódios febris na síndrome são o interferon gama, TNF alfa e IL-6, existindo a possibilidade de uma resposta imune anormal contra micro-organismos comensais da região tonsilar e mucosa oral. ${ }^{5}$ Porém, mais estudos são necessários para definir a etiologia da síndrome.

Além da semelhança com as faringotonsilites, a síndrome PFAPA possui outros diagnósticos diferenciais, embora menos frequentes em nosso meio, destacando-se a neutropenia cíclica, febre familiar do mediterrâneo, síndrome de hipergamaglobulinemia $D$ e artrite reumatoide juvenil sistêmica. . $^{6,9,10,41}$

A neutropenia cíclica apresenta episódios febris antes dos cinco anos de idade, com duração semelhante à sindrome PFAPA. Entretanto, a recorrência episódica da febre acarreta em prejuízo pondoestatural nas crianças, o que não ocorre na síndrome PFAPA. Encontramos aftas nas gengivas e esplenomegalia em alguns casos. A linfadenomegalia é incomum. . $^{9,10,15}$ 


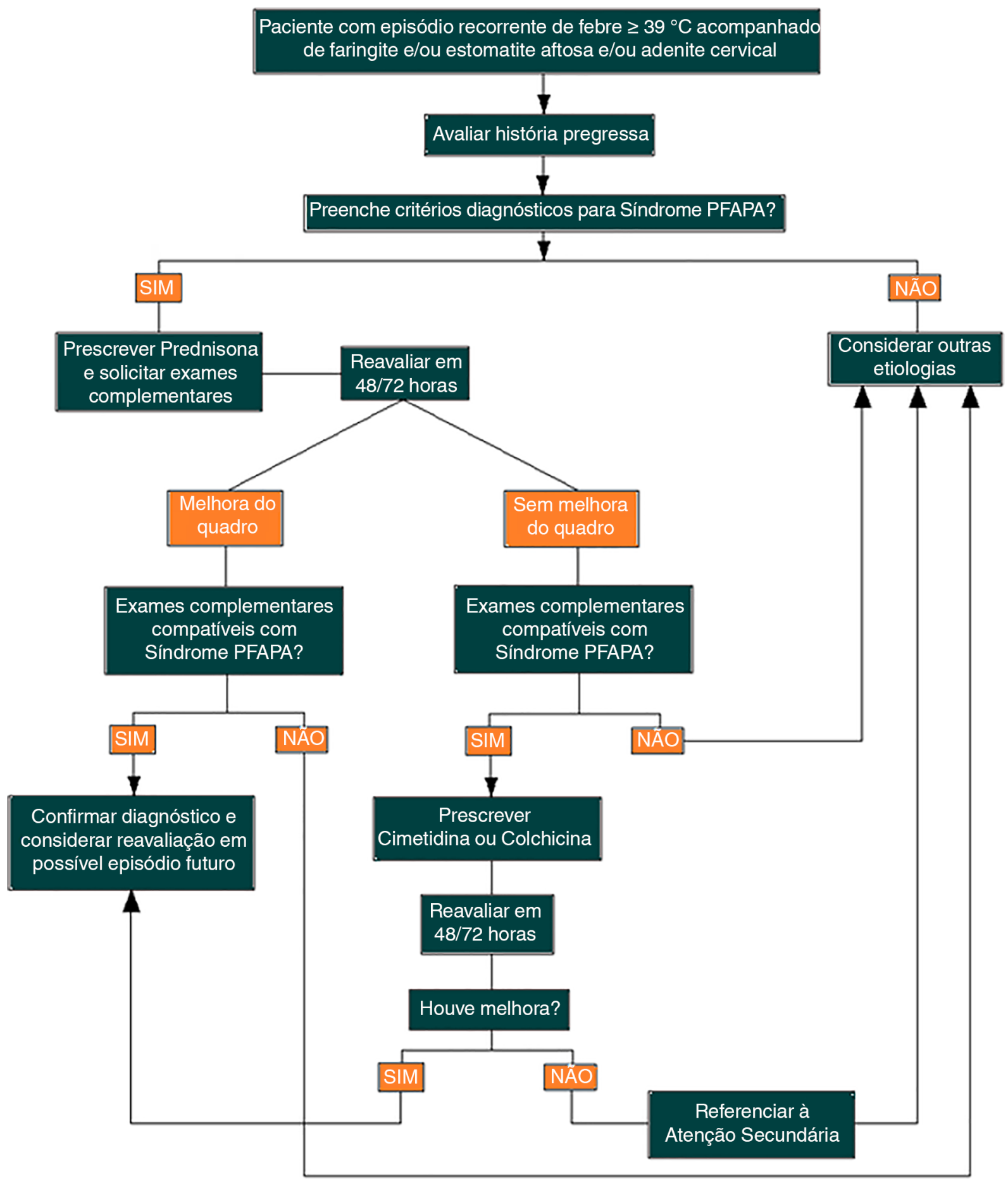

Figura 2. Proposta de fluxograma para diagnóstico e conduta da síndrome PFAPA na Atenção Primária à Saúde

A febre familiar do mediterrâneo acomete, principalmente, turcos, armênios, judeus e árabes. Pode ocorrer após os cinco anos com episódios febris recorrentes e acompanhados de poliserosite, como peritonite e pleurite. Pode haver dor abdominal intensa e manifestações articulares. Entretanto, não encontramos faringite e aftas..$^{9,15}$ 
A síndrome de hipergamaglobulinemia D acomete principalmente crianças de ascendência holandesa. A febre apresenta episódios recorrentes antes de um ano de idade, com pródromos de dor abdominal, diarreia, vômitos e poliartralgia. Pode cursar com exantema petequial ou eritematoso e hepatoesplenomegalia. Durante os episódios febris, os níveis séricos de lgD encontram-se elevados. $6,9,10,15$

A artrite reumatoide juvenil sistêmica apresenta febre com duração de semanas, linfadenopatia generalizada e hepatoesplenomegalia. A avaliação complementar inclui fator reumatoide positivo e Fator Antinuclear (FAN) positivo..$^{9,10,15}$

Assim, quando disponíveis, alguns exames complementares podem auxiliar na exclusão dessas doenças e incluem hemogramas seriados, dosagens de imunoglobulinas séricas $(\lg \mathrm{A}, \lg \mathrm{g}, \lg \mathrm{M}$, $\lg \mathrm{E}$ e $\operatorname{lgD}$ ), Velocidade de Hemossedimentação (VHS), Proteína C Reativa (PCR) e FAN..$^{9,10}$

Os hemogramas ajudam a excluir a neutropenia cíclica, podendo, na síndrome PFAPA, apresentar discreta leucocitose..$^{9,10,15} \mathrm{O}$ VHS é normal e a PCR inferior a $10 \mathrm{mg} / \mathrm{dL}$. O FAN pode ser positivo em cerca de $20 \%$ dos casos, porém, quando negativo, o VHS e a PCR encontram-se aumentados. Além disso, sorologias para citomegalovírus, herpes e vírus Epstein-Barr devem ser realizadas pelo menos em um episódio na suspeita de síndrome PFAPA. ${ }^{9,10}$

Apesar do número de publicações sobre a síndrome PFAPA aumentar ultimamente, a síndrome é pouco conhecida, o que prejudica seu diagnóstico e prolonga a angústia de pacientes. ${ }^{14}$ Por isso, este estudo buscou contribuir com a prática de médicos da atenção primária, divulgando a síndrome PFAPA e seu manejo.

\section{Pontos chave}

1. Cogitar a possibilidade de síndrome PFAPA em todo paciente com episódios febris recorrentes de causa desconhecida;

2. Considerar as diferenças entre as faringotonsilites e a síndrome PFAPA;

3. Respeitar os critérios diagnósticos da síndrome PFAPA;

4. Encaminhar à otorrinolaringologia os pacientes não responsivos à terapêutica e com hipertrofia tonsilar.

\section{Conflito de interesses}

Declaram não haver.

\section{Contribuição do autores}

Concepção e/ou delineamento do estudo: Bueno LA, Paiva DM e Carvalho PHP. Aquisição dos dados: Bueno LA e Paiva DM. Análise e Interpretação dos dados: Bueno LA, Paiva DM e Carvalho PHP.

Redação preliminar: Bueno LA e Paiva DM. Revisão crítica da versão preliminar: Bueno LA, Paiva DM e Carvalho PHP.

Todos os autores aprovaram a versão final e concordaram com prestar contas sobre todos os aspectos do trabalho. 


\section{Agredecimentos}

Agradecemos a Revista Brasileira de Medicina de Família e Comunidade pela oportunidade de divulgação da Síndrome PFAPA e possibilidade de seu manejo por médicos da atenção primária.

\section{Referências}

1. Marshall GS, Edwards KM, Lawton AR. PFAPA syndrome. Pediatr Infect Dis J. 1989;8(9):658-9. Disponível em: https://www.ncbi.nlm. nih.gov/pubmed/2797967

2. Renko M, Salo E, Putto-Laurila A, Saxen H, Mattila PS, Luotonen J, et al. A randomized, controlled trial of tonsillectomy in periodic fever, aphthous stomatitis, pharyngitis, and adenitis syndrome. J Pediatr. 2007;151(3):289-92. https://doi.org/10.1016/j.jpeds.2007.03.015

3. Long SS. Syndrome of Periodic Fever, Aphthous Stomatitis, Pharyngitis, and Adenitis (PFAPA) - what It Isn't. What Is It? J Pediatr. 1999;135(1):1-5. https://doi.org/10.1016/S0022-3476 (99)70316-1.

4. Colotto M, Maranghi M, Durante C, Rossetti M, Renzi A, Anatra MG. PFAPA syndrome in a young adult with a history of tonsillectomy. Intern Med. 2011;50(3):223-5. https://doi.org/10.2169/internalmedicine.50.4421

5. Padeh S, Stoffman N, Berkun Y. Periodic Fever Accompanied by Aphthous Stomatitis, Pharyngitis and Cervical Adenitis Syndrome (PFAPA Syndrome) in Adults. Isr Med Assoc J. 2008;10(5):358-60. Disponível em: https://www.ima.org.il/MedicinelMAJ/viewarticle.asp $x$ ? year $=2008 \&$ month $=05 \&$ page $=358$

6. Fonseca AAR, Cherubini K. Aspectos de interesse clínico sobre a síndrome PFAPA (febre periódica, estomatite aftosa, faringite e adenite). Sci Med. 2005;15(1):68-73. Disponível em: http://revistaseletronicas.pucrs.br/ojs/index.php/scientiamedica/article/view/1546

7. Lantto U, Koivunen P, Tapiainen T, Renko M. Long-Term Outcome of Classic and Incomplete PFAPA (Periodic Fever, Aphthous Stomatitis, Pharyngitis, and Adenitis) Syndrome after Tonsillectomy. J Pediatr. 2016;179:172-7.e1. https://doi.org/10.1016/j.jpeds.2016.08.097

8. Moreira F, Pereira G, Marçal N, Guimarães J, Miranda D. Síndrome PFAPA - Caso clínico. Rev Port Otorrinolaringol Cir Cérvico-Facial. 2002;50(2):165-9. Disponível em: https://www.journalsporl.com/index.php/sporl/article/view/95

9. Silber M. Síndrome PFAPA (Periodic Fever, Adenitis, Pharyngitis, Aphthous Stomatitis) Conhecendo uma Doença Auto-inflamatória. In: Sih T, coord. VI Manual de Otorrinolaringologia Pediátrica da IAPO. Barueri: Gráfica e Editora RR Donnelley; 2007. p. 23-6. Disponível em: http://www.iapo.org.br/novo/secao.asp?s=47

10. Silber M. Síndrome PFAPA (Periodic Fever, Adenitis, Pharyngitis, Aphthous Stomatitis). In: Sih T, coord. IV Manual de Otorrinolaringologia Pediátrica da IAPO. Guarulhos: Lis Gráfica \& Editora; 2005. p. 33-6. Disponível em: http://www.iapo.org.br/novo/secao.asp?s=45

11. Thomas KT, Feder HM Jr, Lawton AR, Edwards KM. Periodic fever syndrome in children. J Pediatr. 1999;135(1):15-21. https://doi. org/10.1016/S0022-3476(99)70321-5

12. Lawton A. Periodic fever with aphthous stomatitis, pharyngitis and adenitis (PFAPA syndrome). 2008. Disponível em: https://www.uptodate. $\mathrm{com} / \mathrm{pt} / \mathrm{home}$

13. Tasher D, Somekh E, Dalal I. PFAPA syndrome: new clinical aspects disclosed. Arch Dis Child. 2006;91(12):981-4.https://doi.org/10.1136/ adc.2005.084731

14. De Cunto C, Britos M, Eymann A, Deltetto N, Liberatore D. Fiebre periódica: descripción de doce pacientes con fiebre periódica, estomatitis aftosa, faringitis y adenitis cervical. Arch Argent Pediatr. 2010;108(5):445-8. Disponível em: http://www.scielo.org.ar/scielo. php?script=sci_arttext\&pid=S032500752010000500011\&lng=es

15. Sampaio I, Marques JG. Síndrome PFAPA (Febre Periódica, Estomatite Aftosa, Faringite, Adenite) Análise Retrospectiva de 21 Casos. Acta Med Port. 2011;24(1):37-42. Disponível em: https://actamedicaportuguesa.com/revista/index.php/amp/article/download/345/115

16. Rigante D, Vitale A, Natale MF, Lopalco J, Andreozzi A, Frediani B, et al. A comprehensive comparison between pediatric and adult patients with periodic fever, aphthous stomatitis, pharyngitis, and cervical adenopathy (PFAPA) syndrome. Clin Rheumatol. 2016;36(2):463-8. https://doi.org/10.1007/s10067-016-3317-7

17. Feder HM, Salazar JC. A clinical review of 105 patients with PFAPA (a periodic fever syndrome). Acta Paediatrica. 2010;99(2):178-84. https://doi.org/10.1111/j.1651-2227.2009.01554.x 
18. Król P, Böhm M, Sula V, Dytrych P, Katra R, Němcová D, et al. PFAPA syndrome: clinical characteristics and treatment outcomes in a large single-centre cohort. Clin Exp Rheumatol. 2013;31(6):980-7. Disponível em: http://www.clinexprheumatol.org/abstract.asp?a=6632

19. Hofer M, Pillet P, Cochard MM, Berg S, Krol P, Kone-Paut I, et al. International periodic fever, aphthous stomatitis, pharyngitis, cervical adenitis syndrome cohort: description of distinct phenotypes in 301 patients. Rheumatology (Oxford). 2014;53(6):1125-9. https://doi. org/10.1093/rheumatology/ket460

20.Scattoni R, Verrotti A, Rinaldi VE, Paglino A, Carelli A, D’Alonzo R. Genital ulcer as a new clinical clue to PFAPA syndrome. Clin Exp Rheumatol. 2014;40(3):286-8. http://dx.doi.org/10.1111/ced.12524

21. Khodaghalian B, Tewary KK, Narchi H. PFAPA with facial swelling- a new association? Indian J Pediatr. 2013;80(5):425-7. https://doi. org/10.1007/s12098-012-0744-6

22. Li CM, Wang CC, Lai CC, Fan HC, Huang WH, Cheng SN. Genital ulcers as an unusual sign of periodic fever, aphthous stomatitis, pharyngotonsillitis, cervical adenopathy syndrome: a novel Symptom? Pediatr Dermatol. 2011;28(3):290-4.https://doi.org/10.1111/j.15251470.2010.01247.x

23. Cantarini L, Vitale A, Bartolomei B, Galeazzi M, Rigante D. Diagnosis of PFAPA syndrome applied to a cohort of 17 adults with unexplained recurrent fevers. Clin Exp Rheumatol. 2012;30(2):269-71. Disponível em: http://www.clinexprheumatol.org/abstract.asp?a=5337

24. Førsvoll J, Kristoffersen EK, Øymar K. Incidence, clinical characteristics and outcome in Norwegian children with periodic fever, aphthous stomatitis, pharyngitis and cervical adenitis syndrome; a population-based study. Acta Pædiatr.2013;102(2):187-92. https://doi.org/10.1111/ apa. 12069

25. Shi T. Faringotonsilites Agudas. In: Sih T, coord. VI Manual de Otorrinolaringologia Pediátrica da IAPO. 6ª ed. Barueri: Gráfica e Editora RR Donnelley; 2007. p. 55-64. Disponível em: http://www.iapo.org.br/novo/secao.asp?s=47

26. Vanoni F, Theodoropoulou K, Hofer M. PFAPA syndrome: a review on treatment and outcome. Pediatr Rheumatol. 2016;14(1):38. https://doi.org/10.1186/s12969-016-0101-9

27. Butbul AvielY, Tatour S, Gershoni Baruch R, Brik R. Colchicine as a therapeutic option in periodic fever, aphthous stomatitis, pharyngitis, cervical adenitis (PFAPA) syndrome. Semin Arthritis Rheum. 2016;45(4):471-4. https://doi.org/10.1016/j.semarthrit.2015.07.005

28. Ciriza Barea E, Gonzalo Herrera NN, Galardi Andonegui MS, Leoz Oses P. Faringoamigdalitis de repetición sin causa infecciosa: síndrome PFAPA. A propósito de un caso. Acta Pediatr Esp. 2011;69(3):140-2. Disponível em: http://www.actapediatrica.com/index.php/secciones/ notas-clinicas/36-faringoamigdalitis-de-repetici\%C3\%B3n-sin-causa-infecciosa-s\%C3\%ADndrome-pfapa-a-prop\%C3\%B3sito-de-uncaso\#.WOTPI9VKjIU

29. Wurster VM, Carlucci JG, Feder HM Jr, Edwards KM. Long-term follow-up of children with periodic fever, aphthous stomatitis, pharyngitis, and cervical adenitis syndrome. J Pediatr. 2011;159(6):958-64. https://doi.org/10.1016/j.jpeds.2011.06.004

30. Dusser P, Hentgen V, Neven B, Koné-Paut I. Is colchicine an effective treatment in periodic fever, aphtous stomatitis, pharyngitis, cervical adenitis (PFAPA) syndrome? Joint Bone Spine. 2016;83(4):406-11. https://doi.org/10.1016/j.jbspin.2015.08.017

31. Tasher D, Stein M, Dalal I, Somekh E. Colchicine prophylaxis for frequent periodic fever, aphthous stomatitis, pharyngitis and adenitis episodes. Acta Paediatr. 2008;97(8):1090-2. https://doi.org/10.1111/j.1651-2227.2008.00837.x

32. Stagi S, Bertini F, Rigante D, Falcini F. Vitamin D levels and effects of vitamin D replacement in children with periodic fever, aphthous stomatitis, pharyngitis, and cervical adenitis (PFAPA) syndrome. Int J Pediatr Otorhinolaryngol. 2014;78(6):964-8. https://doi.org/10.1016/j. ijporl.2014.03.026

33. Licameli G, Lawton M, Kenna M, Dedeoglu F. Long-term surgical outcomes of adenotonsillectomy for PFAPA syndrome. Arch Otolaryngol Head Neck Surg. 2012;138(10):902-6. https://doi.org/10.1001/2013.jamaoto.313

34. Erdogan F, Kulak K, Öztürk O, İpek ï̈, Ceran Ö, Seven H. Surgery vs medical treatment in the management of PFAPA syndrome: a comparative trial. Paediatr Int Child Health. 2016;36(4):270-4. https://doi.org/10.1179/2046905515Y.0000000051

35. Garavello W, Pignataro L, Gaini L, Torretta S, Somigliana E, Gaini R. Tonsillectomy in children with periodic fever with aphthous stomatitis, pharyngitis, and adenitis syndrome. J Pediatr. 2011;159(1):138-42. https://doi.org/10.1016/j.jpeds.2010.12.014

36. Pignataro L, Torretta S, Pietrogrande MC, Dellepiane RM, Pavesi P, Bossi A, et al. Outcome of tonsillectomy in selected patients with PFAPA syndrome. Arch Otolaryngol Head Neck Surg. 2009;135(6):548-53. https://doi.org/10.1001/archoto.2009.56

37. Garavello W, Romagnoli M, Gaini RM. Effectiveness of adenotonsillectomy in PFAPA syndrome: a randomized study. J Pediatr. 2009;155(2):250-3. https://doi.org/10.1016/j.jpeds.2009.02.038 
38. Licameli G, Jeffrey J, Luz J, Jones D, Kenna M. Effect of adenotonsillectomy in PFAPA syndrome. Arch Otolaryngol Head Neck Surg. 2008;134(2):136-40. https://doi.org/10.1001/archoto.2007.7

39. Wong KK, Finlay JC, Moxham JP. Role of Tonsillectomy in PFAPA Syndrome. Arch Otolaryngol Head Neck Surg. 2008;134(1):16-9. https:// doi.org/10.1001/archoto.2007.15

40. Renko M, Salo E, Putto-Laurila A, Saxen H, Mattila PS, Luotonen J, et al. A randomized, controlled trial of tonsillectomy in periodic fever, aphthous stomatitis, pharyngitis, and adenitis syndrome. J Pediatr. 2007;151(3):289-92. https://doi.org/10.1016/j.jpeds.2007.03.015

41. Pinto A, Lindemeyer RG, Sollecito TP. The PFAPA syndrome in oral medicine: differential diagnosis and treatment. Oral Surg Oral Med Oral Pathol Oral Radiol Endod. 2006;102(1):35-9. https://doi.org/10.1016/j.tripleo.2005.12.023 